\title{
Disturbing nocturnal activities
}

\author{
Richard W. Norman, MD, FRCSC
}

See related article on page 604

$\mathrm{N}$ octuria is one of the most common complaints among patients who are evaluated for voiding dysfunction. Their main concern is the inability to return quickly to sleep and the resulting cumulative loss of sleep for them and their spouses.

There is great variability in how patients cope with these unwanted visits to the bathroom. Some experience 1 episode per night and others have 5-6 episodes. The self-study by Kaye ${ }^{1}$ addresses the role of different sedatives and analgesics compared with placebo in terms of ability to reduce the number of nocturic episodes in 1 individual. The baseline nocturia was mild: 1-2 times per night. The number of episodes was reduced from a mean of 1.6 episodes per night with placebo to 0.7 episodes with naproxen and 0.6 with oxazepam $(p<0.05$ for both). It was found that naproxen worked by reducing overnight sodium excretion and urine volumes; oxazepam improved bladder volumes probably by a direct effect on the bladder or its nerve supply. This man also had urgency and, rarely, urgency incontinence, which suggests an element of detrusor overactivity; however, he received benefit from both drugs.

A previous population-based study of Canadian men revealed that men over the age of 50 years get up to void twice per night, on average. ${ }^{2}$ Many patients are reassured by these data. Careful questioning and clinical evaluation can also assure them that there is no suspicion of prostate cancer. These points often elicit the response that the 1-2 episodes of nocturia are not really problematic and that no intervention is required. It would be interesting to conduct a trial of naproxen and oxazepam in patients experiencing more severe nocturia to determine whether the same benefit exists.

In patients for whom nocturia is still a significant bother for them or their spouses, nonpharmacological suggestions such as a fluid restriction, especially alcohol and drinks containing caffeine, during the evening can lead to a substantial improvement in sleep and reduced nocturia. Similarly, elevation of the legs for 30-60 minutes before retiring is useful in mobilizing even the mild day-to-day pedal edema of venous insufficiency and voiding it out before retiring. Patients taking diuretics should be encouraged to take them in the morning. Such behavioural approaches should be tried before any drugs are prescribed.

I was a little surprised at the choice of drugs compared in Kaye's study ${ }^{1}$ because many of the traditional drugs used for nocturia, such as anticholinergics, desmopressin and $\alpha$-blockers, were missing. To fully assess the benefit of naproxen and oxazepam, this comparison will need to be made.

Since there was a benefit from a single dose of naproxen and oxazepam, one wonders if further benefit could be obtained by repetitive dosing of either drug, either separately or in combination. This also raises the question of potential side effects of these specific drugs in this patient population. Naproxen is a nonsteroidal anti-inflammatory drug (NSAID) that inhibits both the COX-1 and COX-2 enzymes and can cause gastrointestinal upset and hypertension from kidney damage or sodium retention. Oxazepam is a benzodiazepine that enhances the effects of $\gamma$-aminobutyric acid on the brain and nerves to produce a calming effect; it can cause drowsiness and dizziness, especially in elderly people.

Both naproxen and oxazepam provided a reduction in nocturia in Kaye's study. ${ }^{1}$ Although this is an innovative use for both of these drugs, there are many questions that must be answered before either could be recommended for routine use. Further assessment with more symptomatic patients of both sexes and evaluation of drug efficacy and safety in combination with standard behavioural interventions are needed.

Professor, Department of Urology, Dalhousie University, Halifax, NS

Competing interests: None declared.

\section{References}

1. Kaye M. Nocturia: a blinded, randomized, parallel placebo-controlled self-study of the effect of 5 different sedatives and analgesics. Can Urol Assoc 1 2008;2:604-8.

2. Norman RW, Nickel CJ, Fish D, et al. "Prostate-related symptoms" in Canadian men 50 years of age and older: prevalence and relationships among symptoms. Br J Urol 1994;74:542-50.

Correspondence: Dr. Richard W. Norman, Suite 620, 5991 Spring Garden Rd., Halifax NS B3H 1Y6; richard.norman@dal.ca 